\title{
Discovery of an old nova shell surrounding the cataclysmic variable V1315 Aql
}

\author{
D. I. Sahman, ${ }^{1 \star}$ V. S. Dhillon, ${ }^{1,2}$ S. P. Littlefair ${ }^{1}$ and G. Hallinan ${ }^{3}$ \\ ${ }^{1}$ Department of Physics and Astronomy, University of Sheffield, Sheffield S3 7RH, UK \\ ${ }^{2}$ Instituto de Astrofisica de Canarias, E-38205 La Laguna, Tenerife, Spain \\ ${ }^{3}$ California Institute of Technology, 1200 East California Boulevard, Pasadena, CA 91125, USA
}

Accepted 2018 April 12. Received 2018 March 29; in original form 2018 March 19

\begin{abstract}
Following our tentative discovery of a faint shell around V1315 Aql reported in Sahman et al., we undertook deep $\mathrm{H} \alpha$ imaging and intermediate-resolution spectroscopy of the shell. We find that the shell has its geometric centre located on V1315 Aql. The mass, spectral features, and density of the shell are consistent with other nova shells, rather than planetary nebulae or supernova remnants. The radial velocity of the shell is consistent with the systemic velocity of V1315 Aql. We believe this evidence strongly suggests that the shell originates from an earlier nova event. This is the first nova shell discovered around a nova-like and supports the theory of nova-induced cycles in mass transfer rates (hibernation theory) first proposed by Shara et al.
\end{abstract}

Key words: novae, cataclysmic variables - stars:individual:V1315 Aql.

\section{INTRODUCTION}

Cataclysmic variables (CVs) are close binary systems in which a white dwarf (WD) primary accretes material from a late-type secondary star, via Roche lobe overflow (see Warner 1995 for a review). Non-magnetic CVs are classified into three main sub-types - the novae, the dwarf novae, and the nova-likes. The novae are defined as systems in which only a single nova eruption has been observed. Novae eruptions have typical amplitudes of 10 mag and are believed to be due to the thermonuclear runaway of hydrogenrich material accreted on to the surface of the white dwarf. The dwarf novae (DNe) are defined as systems which undergo quasiregular (on time-scales of weeks-months) outbursts of much smaller amplitude (typically 6 mag). Dwarf novae outbursts are believed to be due to instabilities in the accretion disc causing it to collapse on to the white dwarf. The nova-like variables (NLs) are the non-eruptive $\mathrm{CVs}$, i.e. objects which have never been observed to show novae or dwarf novae outbursts. The absence of dwarf novae outbursts in NLs is believed to be due to their high mass-transfer rates, producing ionized accretion discs in which the disc-instability mechanism that causes outbursts is suppressed (Osaki 1974); the mass transfer rates in NLs are $\dot{M} \sim 10^{-9} \mathrm{M}_{\odot} \mathrm{yr}^{-1}$ whereas DNe have rates of $\dot{M} \sim 10^{-11} \mathrm{M}_{\odot} \mathrm{yr}^{-1}$ (Warner 1995).

Our understanding of $\mathrm{CV}$ evolution has made great strides in recent years (e.g. Knigge 2010; Knigge, Baraffe \& Patterson 2011). However, one of the main unsolved problems in $\mathrm{CV}$ evolution is: how can the different types of $\mathrm{CV}$ co-exist at the same orbital period? Theory predicts that all CVs evolve from longer to shorter orbital

* E-mail: d.sahman@sheffield.ac.uk periods on time-scales of gigayears, and as they do so the masstransfer rate also declines. At periods longer than approximately $5 \mathrm{~h}$, all CVs should have high mass-transfer rates and appear as novalikes, whereas below this period the lower mass-transfer rate allows the disc-instability mechanism to operate and all CVs should appear as dwarf novae (Knigge et al. 2011). This theoretical expectation, however, is in stark contrast to observations, which show that novalikes are far more common than dwarf novae in the 3-4 h period range (Rodríguez-Gil et al. 2007).

Two possible explanations for the coexistence of nova-likes and dwarf novae at the same orbital periods have been proposed, both of which invoke cycles in $\dot{M}$ on time-scales shorter than the gigayear evolutionary time-scale of CVs. The first explanation is that the $\dot{M}$ cycles are caused by irradiation from the accreting WD, which bloats the secondary star and hence increases $\dot{M}$ (e.g. Büning \& Ritter 2004). Knigge et al. (2011) found that irradiation would cause bloating of $<3$ percent above the period gap, leading to modest fluctuations in $\dot{M}$ with time-scales of the order of $10^{6}-10^{9} \mathrm{yr}$, insufficient to explain the full range in $\dot{M}$ that is observed. The second explanation for variable $\dot{M}$ is a nova-induced cycle. Some fraction of the energy released in the nova event will heat up the WD, leading to irradiation and subsequent bloating of the secondary star. Following the nova event, the system would have a high $\dot{M}$ and appear as a NL. As the WD cools, $\dot{M}$ reduces and the system changes to a DN, or even possibly $\dot{M}$ ceases altogether and the system goes into hibernation. Hence CVs are expected to cycle between nova, NL, and DN states, on time-scales of $10^{4}-10^{5} \mathrm{yr}$ (see Shara et al. 1986).

The cyclical evolution of CVs through nova, NL, and DN phases recently received observational support from the discovery that BK Lyn appears to have evolved through all three phases since its likely 
Table 1. V1315 Aql DEIMOS slit positions, sizes, and spectral range coverage. The RA and Dec. positions are for the centres of the slits.

\begin{tabular}{|c|c|c|c|c|c|c|c|}
\hline \multirow[t]{2}{*}{ Slit name } & \multirow{2}{*}{$\begin{array}{l}\text { RA } \\
\text { (deg) }\end{array}$} & \multirow{2}{*}{$\begin{array}{l}\text { Dec. } \\
\text { (deg) }\end{array}$} & \multirow{2}{*}{$\begin{array}{c}\text { Slit } \\
\text { length } \\
(\operatorname{arcsec})\end{array}$} & \multirow{2}{*}{$\begin{array}{c}\text { Slit } \\
\text { position } \\
\text { angle } \\
(\mathrm{deg})\end{array}$} & \multirow{2}{*}{$\begin{array}{c}\text { Slit } \\
\text { width } \\
(\operatorname{arcsec})\end{array}$} & \multicolumn{2}{|c|}{ Wavelength range } \\
\hline & & & & & & Start $(\AA)$ & End $(\AA)$ \\
\hline Blank Sky 1 & 288.5230602 & 12.2217710 & 58.872 & 154.4 & 0.7 & 4780 & 7432 \\
\hline Blank Sky 2 & 288.4896981 & 12.3217610 & 49.423 & 154.4 & 0.7 & 4867 & 7514 \\
\hline Shell 1 & 288.4995975 & 12.3003892 & 32.904 & 154.4 & 1.0 & 4868 & 7521 \\
\hline Shell 2 & 288.5116182 & 12.2922447 & 46.933 & 150.0 & 1.0 & 4915 & 7576 \\
\hline Shell 4 & 288.4507472 & 12.3566674 & 65.419 & 170.0 & 1.0 & 4699 & 7343 \\
\hline Shell 5 & 288.5171327 & 12.2647206 & 59.595 & 154.4 & 1.0 & 4877 & 7529 \\
\hline Shell 6 & 288.4757487 & 12.2611586 & 43.389 & 150.0 & 1.0 & 4614 & 7272 \\
\hline Shell 7 & 288.4367229 & 12.3103574 & 43.386 & 130.0 & 1.0 & 4487 & 7170 \\
\hline V1315 Aql & 288.4769928 & 12.3013719 & 42.173 & 154.4 & 1.0 & 4735 & 7382 \\
\hline Star 1 & 288.5492156 & 12.2172214 & 49.155 & 154.4 & 1.0 & 5058 & 7565 \\
\hline Star 2 & 288.5403078 & 12.2461305 & 45.629 & 154.4 & 1.0 & 5047 & 7592 \\
\hline
\end{tabular}

nova outburst in the year AD 101 (Patterson et al. 2013). A second piece of evidence has come from the discovery of nova shells around the dwarf novae Z Cam and AT Cnc (Shara et al. 2007; Shara et al. 2012), verifying that they must have passed through an earlier nova phase. Shara et al. (2017b) also found a nova shell from Nova Sco 1437 and were able to associate it with a nearby dwarf nova using its proper motion. A more obvious place than DNe to find nova shells is actually around NLs, as the nova-induced cycle theory suggests that the high $\dot{M}$ in NLs is due to a recent nova outburst. Finding shells around NLs would lend further support to the existence of nova-induced cycles and hence why systems with different $\dot{M}$ are found at the same orbital period.

In our earlier paper (Sahman et al. 2015, hereafter S15), we presented the initial results of our search for nova shells around CVs. We reported the tentative discovery of a possible shell around the nova-like V1315 Aql (orbital period $3.35 \mathrm{~h}$ ). We subsequently obtained intermediate-resolution spectroscopy of this shell, in an effort to determine its physical characteristics and to ascertain if it is associated with the nova-like. The results of these spectroscopic observations, along with a more in-depth analysis of the $\mathrm{H} \alpha$ images of the V1315 Aql shell shown in S15, are presented in this paper.

\section{OBSERVATIONS AND DATA REDUCTION}

\subsection{Observations}

\subsubsection{INT images}

We used the Wide Field Camera ${ }^{1}$ at the prime focus of the $2.5 \mathrm{~m}$ Isaac Newton Telescope on La Palma to image V1315 Aql on the night of 2014 August 2. This set up gave a plate scale of 0.33 arcsec pixel $^{-1}$ and a field view of approx. 34 arcmin $\times 34$ arcmin. $\mathrm{H} \alpha$ is generally the strongest feature in the spectra of nova shells, with a velocity width of up to $2000 \mathrm{~km} \mathrm{~s}^{-1}$ (e.g. Duerbeck \& Seitter 1987). In order to maximize the detection of light from the shell and minimize the contribution of sky, we therefore used a narrowband $\left[95 \AA\right.$ full width at half-maximum $(\mathrm{FWHM})=4300 \mathrm{~km} \mathrm{~s}^{-1}$ ] interference filter centred on the rest wavelength of $\mathrm{H} \alpha$ (ING fil- ter number $197^{2}$ ). We took eight $900 \mathrm{~s} \mathrm{H} \alpha$ exposures, with four of the images dithered by \pm 20 arcsec in both RA and Dec. The observing conditions were good throughout the run: the sky was always photometric, there was no evidence of dust and the seeing was 1.5 arcsec.

\subsubsection{Keck DEIMOS spectra}

We used the DEIMOS (Faber et al. 2003) multislit spectrograph on the $10 \mathrm{~m}$ Keck II telescope on Hawaii, on the night of 2015 June 13. We obtained 39 spectra of $300 \mathrm{~s}$ duration each, using the $1200 \mathrm{G}$ grating centred on $6000 \AA$ and the GG455 order-blocking filter. This gave a wavelength coverage of 4550-7500 $\AA$, with an FWHM resolution of $1.6 \AA$. The seeing was $0.7 \operatorname{arcsec}$, and there was some thin cloud present.

The slit mask design requires that the slits cannot overlap in the spatial direction, so we placed seven slits around the edges of the roughly circular shell. We also placed a slit on V1315 Aql itself and chose four nearby stars for flux calibration. We identified two areas of blank sky for sky subtraction. The positions of each slit on the sky are shown in Fig. 2, and full details of the position, orientation, and wavelength coverage of each slit are given in Table 1.

\subsubsection{5 m Telescope - La Palma}

Our Keck spectra included four stars for flux calibration but unfortunately they did not appear in any photometric catalogues. In order to allow us to perform flux calibration, we therefore obtained additional images of the four stars together with two catalogue stars (TYC 1049-408-1 and IPHAS J1911411.93+121357.7) using the $0.5 \mathrm{~m}$ robotic telescope $p t 5 m$ on La Palma (Hardy et al. 2015). The observations were taken on 2016 October 7, when we took four images in each of the $B, V, R$, and $I$ filters with an exposure time of 1 min each, and on 2016 November 18, when we took four $40 \mathrm{~s}$ $R$-band images and four $360 \mathrm{~s} B$-band images.

\footnotetext{
${ }^{1}$ http://www.ing.iac.es/astronomy/instruments/wfc/
}

${ }^{2}$ http://catserver.ing.iac.es/filter/list.php?instrument=WFC 


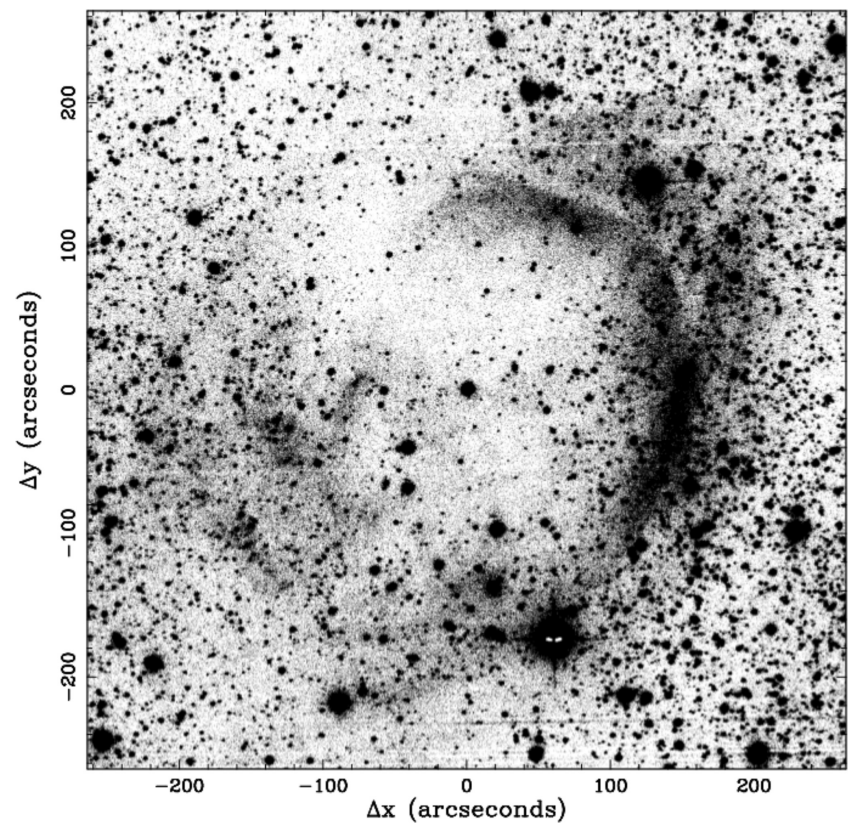

Figure 1. INT WFC H $\alpha$ image of the nova shell around V1315 Aql. The binary is located at the centre of the image. North is up and east is left.

\subsection{Data reduction}

\subsubsection{INT images}

The INT images were debiased using the median level of the overscan strip and flat-fielded using normalized twilight sky flats. All image processing was carried out using THELI. ${ }^{3}$ Fig. 1 shows the final stacked image of the shell.

\subsubsection{Keck DEIMOS spectra}

We used IRAF to reduce the DEIMOS spectra. The spectra were bias corrected using the overscan strip on the chips, and were flatfielded using quartz lamp flats. We had difficulty in performing the background sky subtraction because the two blank sky slits we had chosen both contain small residual $\mathrm{H} \alpha$ emission lines, possibly from the nova shell.

We then tried using the sky portion of our four flux calibration stars, but we found that the spectra of the three closest to the shell (Stars 1-3) also contained low levels of residual $\mathrm{H} \alpha$ emission (see Fig. 5). The best results were obtained with sky from Star 4, which is farthest from the shell and showed negligible $\mathrm{H} \alpha$ emission - this was used for all subsequent background sky subtraction.

\subsection{3 pt5m images}

The images were bias and flat-field corrected using standard IRAF procedures. This allowed us to derive magnitudes for the four flux calibration stars, as shown in Table 2. We also found 2MASS infrared magnitudes (Skrutskies et al. 2006) for Star 2. Hence, the star with the most complete set of magnitudes was Star 2. We input these values into the Virtual Observatory SED Analyser (VOSA - see Bayo et al. 2008) to determine the spectral type of Star 2,

${ }^{3}$ http://www.ing.iac.es/astronomy/instruments/wfc/WFC-THELI-reducti on.html

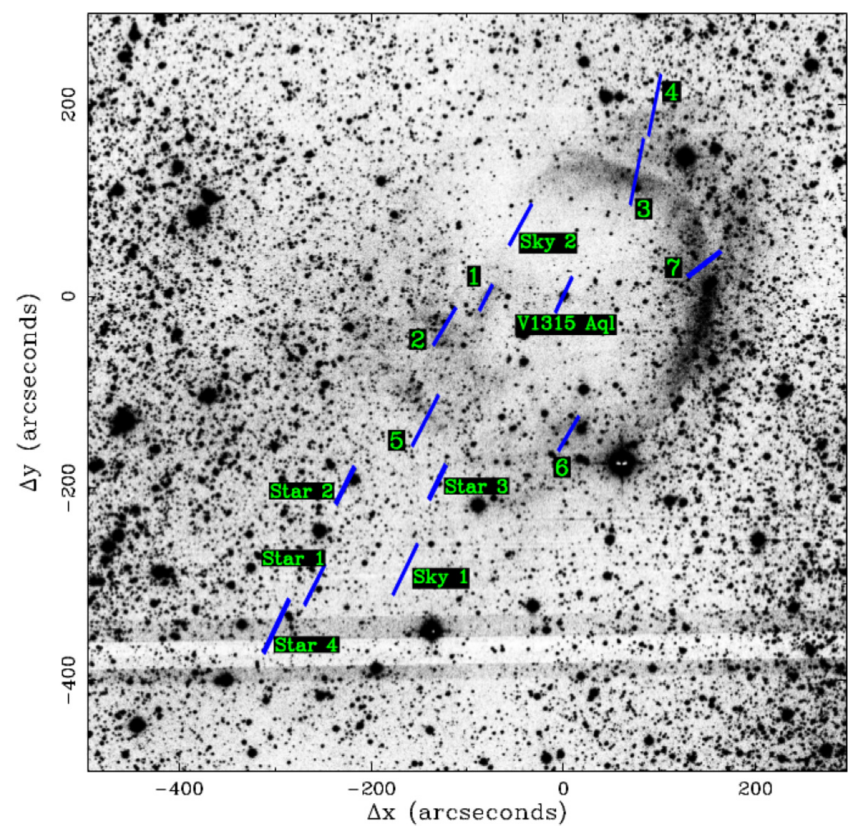

Figure 2. INT WFC H $\alpha$ image of the nova shell around V1315 Aql with the Keck DEIMOS slit positions and sizes overlaid. V1315 Aql is situated at $x=0, y=0$. The seven shell slits are numbered, and the two blank sky slits are also shown. The four flux calibration stars are marked Star 1-4. North is up and east is left. The horizontal band across the image at $y \sim-380$ is the gap between two of the CCDs in the WFC mosaic.

Table 2. Magnitudes of the four flux-calibration stars observed with Keck DEIMOS. The errors on the $B, V, R$, and $I$ magnitudes are \pm 0.3 mag. See Table 1 for the positions of the stars on the sky.

\begin{tabular}{lrrrr}
\hline Band & Star 1 & Star 2 & Star 3 & Star 4 \\
\hline$B$ & 18.8 & 16.5 & - & - \\
$V$ & 17.6 & 15.3 & 19.5 & 18.3 \\
$R$ & 16.9 & 14.5 & 18.4 & 16.5 \\
$I$ & 15.1 & 13.7 & 17.2 & 14.6 \\
2MASS $J$ & - & 12.508 & - & - \\
2MASS $H$ & - & 11.798 & - & - \\
2MASS $K$ & - & 11.646 & - & - \\
\hline
\end{tabular}

obtaining M4V $( \pm 2)$. We then used VOSA to generate a template spectrum of an M4V star, which we used to flux calibrate the Keck spectra in IRAF.

\subsubsection{Review of satellite imagery}

We searched the GALEX $U V$ satellite footprint using the GalexView interface (Bianchi 2014), but no observations were taken of the field around V1315 Aql. We also examined the WISE $22 \mu \mathrm{m}$ data (Wright et al. 2010), and there was no emission in the vicinity of V1315 Aql.

\section{RESULTS}

\subsection{INT image}

The $\mathrm{H} \alpha$ image of the shell surrounding V1315 Aql is shown in Fig. 1. The images clearly show one, possibly two roughly spherical shells centred on V1315 Aql. The lobe towards the west has the most 


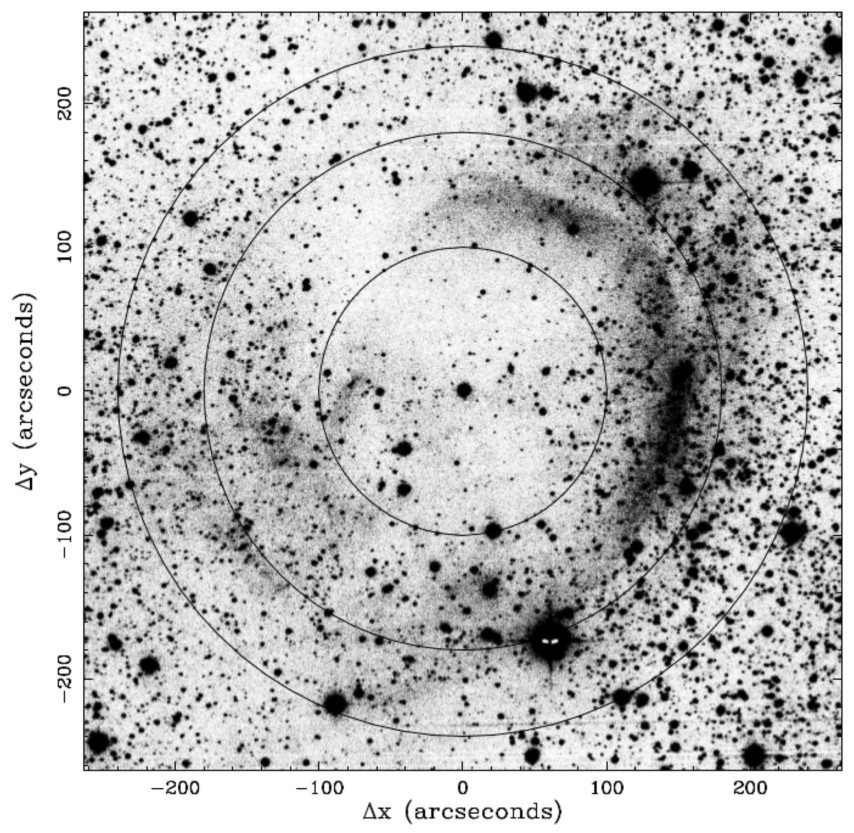

Figure 3. INT WFC H $\alpha$ image of the shell with overlaid circles centred on V1315 Aql of radii 100, 180, and 240 arcsec. North is up and east is left.

prominent emission. There was no evidence of nebulosity on wider scales than shown in Fig. 2.

There is a possibility that the shell is unrelated to V1315 Aql, and it may just be a line-of-sight alignment of a foreground or background cloud of gas in the Milky Way. To determine that the shell does indeed originate from V1315 Aql, we need to determine if it has the same systemic velocity as the binary, and that its composition is comparable to other nova shells, and to rule out other types of nebulosity, e.g. planetary nebulae, supernovae remnants.

\subsection{Geometry of the shell}

In Fig. 3, we show the image of the shell with circles centred on V1315 Aql overlaid. The radii of the circles are 100, 180, and $240 \mathrm{arcsec}$. The inner annulus between 100 and $180 \mathrm{arcsec}$ contains the most prominent areas of emission (from the north around to the west), and appears to be centred on V1315 Aql. The outer annulus also contains a fainter arc of emission to the north-west, and some fainter areas of emission to the south-east, which also appear to be centred on V1315 Aql.

\subsection{Keck DEIMOS spectra}

In Fig. 4, we show the spectrum of V1315 Aql. The spectrum shows strong, broad (FWHM of $\mathrm{H} \alpha$ is $900 \mathrm{~km} \mathrm{~s}^{-1}$ ) Balmer and He I emission lines from the accretion disc. The spectrum is very similar to that shown in Dhillon \& Rutten (1995).

\subsubsection{Emission lines}

The spectra of the seven shell slits and the blank sky slits in the range 6540-6600 $\AA$ are shown in Fig. 5. Note that the blank sky 2 slit spanned two CCDs in the spectrograph and each part is shown separately. The shell spectra all show single-peaked emission lines of $\mathrm{H} \alpha$ and a pair of N[II] lines at 6548 and $6583 \AA$. These lines are

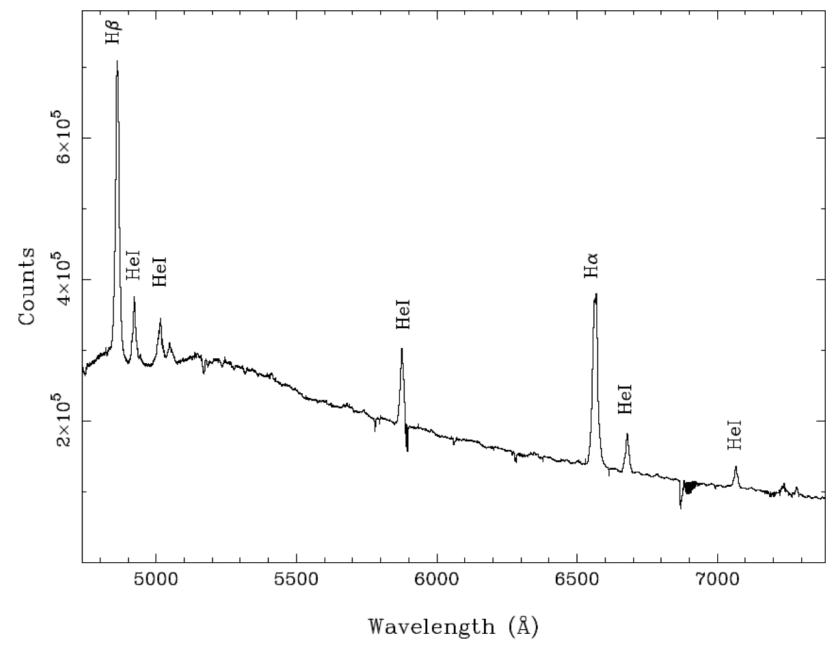

Figure 4. Keck DEIMOS spectrum of V1315 Aql. Note that we did not flux calibrate this spectrum because the flux calibration stars do not cover its whole wavelength range.

characteristic of old nova shells (Downes, Duerbeck \& Delahodde 2001).

We also detected $\mathrm{H} \beta$ in those shell spectra that covered $4861 \AA$. Unfortunately, none of the spectra of the four flux-calibration stars covered this wavelength, and hence we were unable to flux calibrate the $\mathrm{H} \beta$ lines. We also found the $\mathrm{S}[$ пा] 6716 and $6731 \AA$ lines in shell slits 2, 3, 5, 6, and 7, as shown in Fig. 6. The average ratio of the two $\mathrm{S}[\mathrm{II}]$ lines is $1: 1.4$.

We searched for the emission lines N[II] $5755 \AA$, O[I] 6300, 6300, $6364 \AA$ and O[III] 4363, 4959, $5007 \AA$, often seen in nova shell spectra, but none were detected. There are faint lines at 5679 , 5740, and $5742 \AA$, presumably from N I and N II, in shell slits 3 and 4 , but these are not present in any other slits.

There is also $\mathrm{H} \alpha$ emission present in both the sky portions of the slit centred on V1315 Aql, though any N[II] lines present are lost in the noise. We show the $\mathrm{H} \alpha$ line profile from the sky on the south-east side of the V1315 Aql slit in Fig. 7.

The FWHM of the $\mathrm{H} \alpha$ and $\mathrm{N}[\mathrm{II}]$ lines of all the shell slits are listed in Table 3.

\subsubsection{Systemic velocity of V1315 Aql}

Historically the systemic velocity of V1315 Aql, $\gamma$, has been difficult to determine because of the complex behaviour of its disc emission lines and lack of absorption lines from the primary and secondary stars. Downes et al. (1986) presented radial velocity data for $\mathrm{H} \beta, \mathrm{H} \gamma$, and He II $4686 \AA$ emission lines. They derived values for $\gamma$ consistent with zero from the $\mathrm{H} \beta$ and He II $4686 \AA$ lines, but the $\mathrm{H} \gamma$ line gave a value of $100 \mathrm{~km} \mathrm{~s}^{-1}$. Dhillon, Marsh \& Jones (1991) also used the $\mathrm{H} \beta, \mathrm{H} \gamma$, and He II $4686 \AA$ emission lines and the $\mathrm{He}_{\mathrm{I}} 4471 \AA$ line and derived a $\gamma$ range of -4 to $+93 \mathrm{~km} \mathrm{~s}^{-1}$. Given the unreliability of the broad emission lines from the accretion disc to determine $\gamma$, in the following subsection we will use our own measurements of the radial velocity of the shell to determine if they are consistent.

\subsubsection{Radial velocities of shell emission lines}

To measure the radial velocities of the emission lines, we fitted a Gaussian to the $\mathrm{H} \alpha$ line of the shell and measured the wavelength 


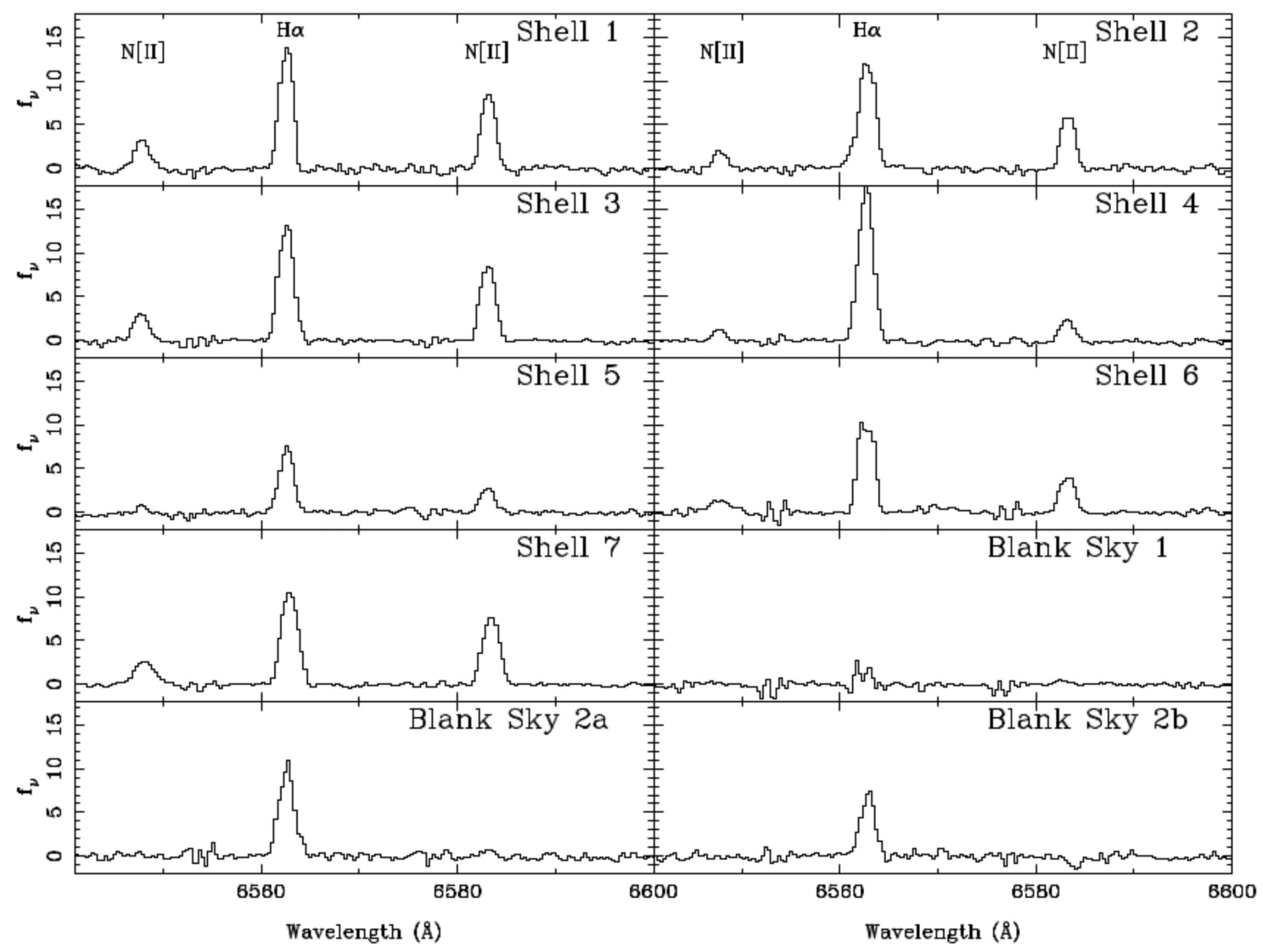

Figure 5. Spectra of the seven shell slits (see Fig. 2), and the blank sky slits from 6540 to $6600 \AA$. The flux is in units of $\mu \mathrm{Jy} \operatorname{arcsec}^{-2}$. The slit spectra all show the presence of $\mathrm{H} \alpha$ and N[II] $6548 \AA$ and N[II] $6583 \AA$. H $\alpha$ is also present in the blank sky slits. The Blank Sky 2 slit fell across two CCDs on the detector and so we show each spectrum separately, as $2 \mathrm{a}$ and $2 \mathrm{~b}$.

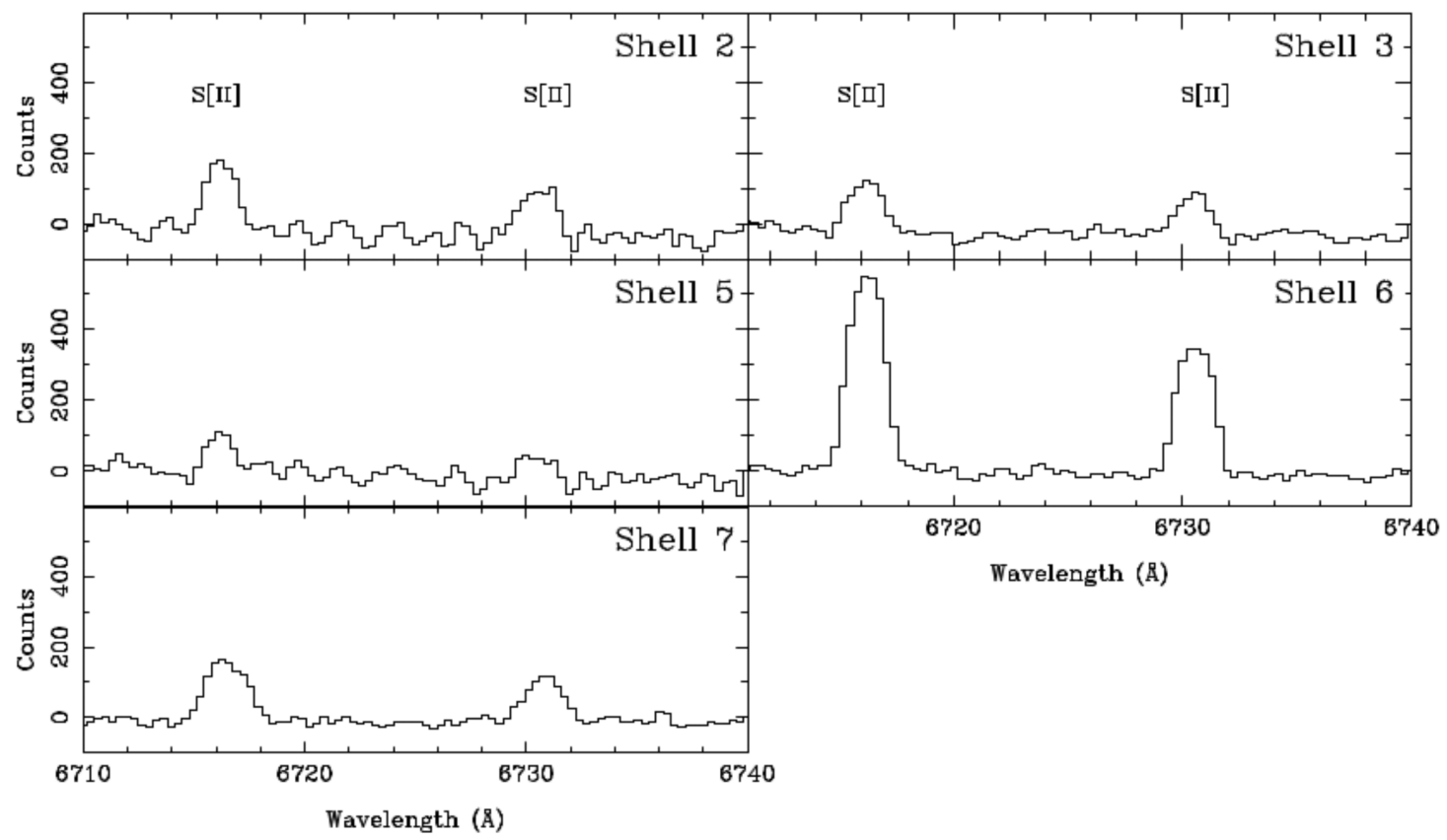

Figure 6. Spectra of the five shell slits from 6710 to $6740 \AA$ showing the S[II] emission lines at 6716 and $6731 \AA$. 


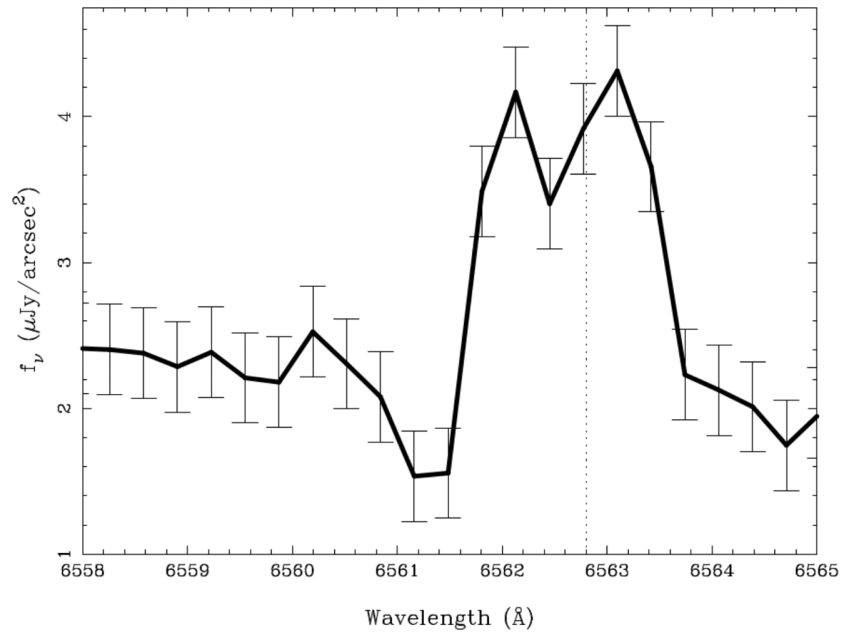

Figure 7. $\mathrm{H} \alpha$ spectrum of the sky from the south-east side of the V1315 Aql slit. The error bars show the noise levels of the background sky.

at the centre of the Gaussian. The resulting shell radial velocities are shown in Table 3.

The spectrum of the sky on the south-east side of the slit centred on V1315 Aql is shown in Fig. 7. The plot shows tentative evidence of a double-peaked structure. We measured the radial velocity of each peak to be -33 and $14 \mathrm{~km} \mathrm{~s}^{-1}$. If we assume that the two peaks represent emission from the front and back sides of a spherically expanding shell, then the average of the two gives a systemic velocity of $\gamma \approx-10 \mathrm{~km}^{-1}$, and an expansion velocity of $\sim 25 \mathrm{~km} \mathrm{~s}^{-1}$. We analysed the sky on the north-east side of V1315 Aql and it too showed a double-peaked structure, although it is less pronounced.

The seven shell slits were placed at the edges of the shell. The expansion velocity of the edge of the shell will be tangential to the line of sight and will not affect the radial velocities, which should be similar to the overall systemic velocity. The measured shell radial velocities are shown in Table 3 and are broadly comparable with the systemic velocity of $-10 \mathrm{~km} \mathrm{~s}^{-1}$ derived above, apart from shells 6 and 7 which differ by 14 and $16 \mathrm{~km} \mathrm{~s}^{-1}$, respectively.

The Galactic velocity of V1315 Aql relative to the Sun can be derived from its Galactic coordinates, $l=46.4, b=0^{\circ}$, which give a radial velocity of $7 \mathrm{~km} \mathrm{~s}^{-1}$. This is broadly consistent with the systemic velocity derived above.

\subsubsection{Line fluxes}

The fluxes of the emission lines from the shell in each of the slits are given in Table 4. Assuming a shell radius of 220 arcsec, a distance of $489 \mathrm{pc}$ (Ak et al. 2008), and using the $\mathrm{H} \alpha$ flux from each slit, we can estimate the total flux from the whole shell. However, we can see by examining Fig. 3 that the shell is fragmented and clumpy and only a small fraction is actually emitting. If we assume that 10 per cent of the full shell is emitting and take an average $\mathrm{H} \alpha$ flux from the seven shell slits of $1.70 \times 10^{-17} \mathrm{erg} \mathrm{cm}^{-2} \mathrm{~s}^{-1} \operatorname{arcsec}^{-2}$, we obtain a total $\mathrm{H} \alpha$ luminosity of $7.1 \times 10^{30} \mathrm{erg} \mathrm{s}^{-1}$.

The plots of Downes et al. (2001) showing the temporal reduction in the $\mathrm{H} \alpha$ luminosities of shells from fast and slow novae have a lower limit of $\log L=30$ at $100 \mathrm{yr}$. We note that the source of the V1315 Aql luminosity is likely to include emission from shock interaction with pre-existing ISM. This would enhance the flux, and lead to an underestimate of the age of the shell. We conclude that the shell is likely to be significantly older than $100 \mathrm{yr}$.

\subsubsection{Time of nova eruption}

The distance to V1315 Aql was measured by Ak et al. (2008) as $489 \pm 49$ pc, computed from the period-luminosity-colours (PLCs) relation of $\mathrm{CV}$ s calibrated with $2 M A S S$ photometric data. The angular radius of the shell on our image is $\sim 4$ arcmin, giving a physical radius of $1.7 \times 10^{13} \mathrm{~km}$. Duerbeck \& Seitter (1987) found that the velocity of nova shells reduces by half every 50-100 yr. Using our measured expansion velocity of $\sim 25 \mathrm{~km} \mathrm{~s}^{-1}$, and assuming an initial velocity of $2000 \mathrm{~km} \mathrm{~s}^{-1}$ (see table 8.1 in Bode \& Evans 2008), we estimate that the nova explosion occurred $\sim 500-600 \mathrm{yr}$ ago. However, if we take more extreme values for the initial ejection velocity, say $700 \mathrm{~km} \mathrm{~s}^{-1}$ and a deceleration half-life of $200 \mathrm{yr}$ then the age of the nova increases to $1200 \mathrm{yr}$.

Assuming that the visual magnitude of V1315 Aql was the same prior to the nova event as it is now, $m_{V}=14.3$, and taking the average brightening of a nova to be $\sim 11$ mag (Bode \& Evans 2008), the system would have been at $m_{V} \sim 3.3$ at peak brightness, clearly visible to the naked eye. Novae decline rapidly and so it would have dropped below the naked-eye visibility limit of $m_{V} \sim 6$ within a few days. We reviewed the catalogues of ancient Chinese and Asian novae and supernovae sightings by Stephenson (1976) which include sightings from $532 \mathrm{BC}$ up to $1604 \mathrm{AD}$. We could find no record of an event close to the coordinates of V1315 Aql. If the nova eruption occurred when V1315 Aql was close to the Sun in the sky, and it was brighter than $m_{V} \sim 6$ for only a few days, it may well have been hidden in twilight and hence gone unnoticed.

\subsubsection{Temperature and density of the shell}

The method most often used to determine the temperature and density of gaseous nebulae is to measure the ratio of the intensities of particular emission lines from the same species of ions. Two ions which are often used are $\mathrm{N}[\mathrm{II}]$ and $\mathrm{O}[\mathrm{III}]$. We were unable to detect any $\mathrm{O}[\mathrm{III}]$ lines in our spectra, and the $\mathrm{N}[\mathrm{II}]$ ratio requires a flux measurement of the $5755 \AA$ line, which we were only able to detect very weakly in shell slit 1 . It was not present in any other slit. Hence we can only place an upper limit on the electron temperature $\left(T_{\mathrm{e}}\right)$ of the shell of $5000 \mathrm{~K}$ using fig. 5.1 from Osterbrock (1989).

\subsubsection{Mass of the shell}

We can derive a rough estimate of the mass of the shell using the technique set out in Corradi et al. (2015). They derived the ionized hydrogen masses of several planetary nebulae using the formula

$m_{\text {shell }}\left(\mathrm{H}^{+}\right)=\frac{4 \pi D^{2} F(\mathrm{H} \beta) m_{\mathrm{p}}}{h \nu_{\mathrm{H} \beta} n_{\mathrm{e}} \alpha_{\mathrm{H} \beta}^{\mathrm{eff}}\left(H^{0}, T_{\mathrm{e}}\right)}$,

where $D$ is the distance to the object, $F(\mathrm{H} \beta)$ is the $\mathrm{H} \beta$ flux, $m_{\mathrm{p}}$ is the mass of a proton, $h v_{\mathrm{H} \beta}$ is the energy of an $\mathrm{H} \beta$ photon, $n_{\mathrm{e}}$ is the electron density $\mathrm{cm}^{-3}$, and $\alpha_{\mathrm{H} \beta}^{\text {eff }}\left(H^{0}, T_{\mathrm{e}}\right)$ is the effective recombination coefficient for $\mathrm{H} \beta$. This formula is also applicable to nova shells (Osterbrock 1989).

As we pointed out in Section 3.3.1, the spectra of our four flux calibration stars do not cover $\mathrm{H} \beta$ so we are unable to derive a flux directly. However, we can make a rough estimate as follows. The $\mathrm{H} \beta$ line is present in four shell slits (Nos. 3, 4, 6, and 7). We can measure the counts for both $\mathrm{H} \alpha$ and $\mathrm{H} \beta$. The DEIMOS exposure time calculator for a source that is flat in frequency gives the ratio of counts for $\mathrm{H} \alpha: \mathrm{H} \beta$ as approximately $1: 0.3$. Assuming that 10 per cent of the full shell is emitting and taking an average $\mathrm{H} \alpha$ flux from the seven shell slits of $1.70 \times 10^{-17} \mathrm{erg} \mathrm{cm}^{-2} \mathrm{~s}^{-1} \operatorname{arcsec}^{-2}$, 
Table 3. The first column shows the radial velocities in $\mathrm{km} \mathrm{s}^{-1}$ of the $\mathrm{H} \alpha$ emission line in the spectra of the seven V1315 Aql shell slits and the sky portion of the four flux calibration stars. The errors on the velocities are $\pm 5 \mathrm{~km} \mathrm{~s}^{-1}$. The other columns show the FWHM in km s${ }^{-1}$ of each line. The errors on the FWHM are $\pm 8 \mathrm{~km} \mathrm{~s}^{-1}$. The $\mathrm{H} \alpha$ line in the V1315 Aql sky has a double peak and both radial velocities are shown. All values and errors were obtained from Gaussian fits to the emission lines.

\begin{tabular}{|c|c|c|c|c|c|c|c|c|c|c|}
\hline Slit name & $\begin{array}{r}\text { Radial } \\
\text { velocity }\end{array}$ & $\mathrm{H} \alpha$ & $\begin{array}{r}\mathrm{N}[\mathrm{II}] \\
6548 \AA\end{array}$ & $\begin{array}{r}\mathrm{N}[\mathrm{II}] \\
6583 \AA\end{array}$ & $\mathrm{H} \beta$ & $\begin{array}{r}\mathrm{N}_{\mathrm{I}} \\
5676 \AA\end{array}$ & $\begin{array}{r}\mathrm{N}_{\text {II }} \\
5740 \AA\end{array}$ & $\begin{array}{r}\mathrm{N}_{\mathrm{I}} \\
5742 \AA\end{array}$ & $\begin{array}{r}S_{\text {II }} \\
6716 \AA\end{array}$ & $\begin{array}{r}S_{\text {II }} \\
6731 \AA\end{array}$ \\
\hline Shell 1 & -8 & 71 & 82 & 72 & 0 & 0 & 0 & 0 & 0 & 0 \\
\hline Shell 3 & -5 & 80 & 79 & 79 & 122 & 143 & 206 & 0 & 68 & 68 \\
\hline Shell 4 & -3 & 79 & 59 & 75 & 89 & 78 & 0 & 124 & 0 & 0 \\
\hline Shell 5 & -9 & 75 & 54 & 75 & 0 & 0 & 0 & 0 & 52 & 64 \\
\hline Shell 6 & -26 & 84 & 87 & 80 & 0 & 0 & 0 & 0 & 78 & 76 \\
\hline V1315 Aql & -33 and 14 & 0 & 0 & 0 & 0 & 0 & 0 & 0 & 0 & 0 \\
\hline \multicolumn{11}{|l|}{ Sky } \\
\hline Star 1 & 11 & - & - & - & - & - & - & - & - & - \\
\hline Star 2 & -23 & - & - & - & - & - & - & - & - & - \\
\hline Star 3 & 29 & - & - & - & - & - & - & - & - & - \\
\hline Star 4 & 14 & - & - & - & - & - & - & - & - & - \\
\hline
\end{tabular}

Table 4. $\mathrm{H} \alpha$ and $\mathrm{N}[\mathrm{II}]$ flux $\left(\mathrm{erg} \mathrm{s}^{-1} \mathrm{~cm}^{-2} \operatorname{arcsec}^{-2} \times 10^{-18}\right)$ from the seven shell slits. The errors on the flux values are \pm 25 per cent.

\begin{tabular}{lrrr}
\hline Shell slit no. & $\mathrm{H} \alpha$ & $\mathrm{N}[\mathrm{II}] 6548 \AA$ & $\mathrm{N}[\mathrm{II}] 6583 \AA$ \\
\hline 1 & 17.2 & 4.22 & 10.8 \\
2 & 18.1 & 2.10 & 7.64 \\
3 & 18.3 & 4.16 & 11.8 \\
4 & 24.2 & 1.47 & 3.03 \\
5 & 9.58 & 0.73 & 3.62 \\
6 & 15.4 & 2.64 & 5.61 \\
7 & 16.2 & 4.80 & 11.9 \\
\hline
\end{tabular}

we obtain a total $\mathrm{H} \alpha$ flux of $2.49 \times 10^{-13} \mathrm{erg} \mathrm{cm}^{-2} \mathrm{~s}^{-1}$ from the whole shell allowing us to derive an $\mathrm{H} \beta$ flux of $F(\mathrm{H} \beta)=8.9 \times 10^{-14}$ erg $\mathrm{cm}^{-2} \mathrm{~s}^{-1}$.

The electron density, $n_{e}$, can be estimated using the S[II] 6716 and 6731 line ratio, which we found to be 1.4 (see Section 3.3.1). Fig. 5.8 in Osterbrock (1989) shows the electron density versus intensity ratio at $T_{\mathrm{e}}=10000 \mathrm{~K}$ and indicates a scaling of $n_{e}\left(10^{4} / T_{\mathrm{e}}\right)^{1 / 2}$. We found a maximum temperature of $5000 \mathrm{~K}$ which gives an electron density of $\sim 22 \mathrm{~cm}^{-3}$.

Finally, using the distance measured by Ak et al. (2008) of $489 \mathrm{pc}$ and a value for $\alpha_{\mathrm{H} \beta}^{\mathrm{eff}}\left(H^{0}, T_{\mathrm{e}}\right)$ of $3.78 \times 10^{-14}$, for Case A conditions at $T_{\mathrm{e}}=5000 \mathrm{~K}$ listed in table 4.1 of Osterbrock (1989) gives a maximum mass of

$m_{\text {shell }}\left(H^{+}\right) \simeq 2 \times 10^{-4} \mathrm{M}_{\odot}$.

There is no need to correct for extinction as Rutten, van Paradijs \& Tinbergen (1992) found $E(B-V)=0$ for V1315 Aql using IUE spectra of interstellar absorption bands around $2200 \AA$. In view of the many assumptions used to estimate the mass of the shell, it should be treated as an order of magnitude approximation.

As nova shells expand, they decelerate as they sweep up preexisting circumstellar gas, which leads to a doubling of their mass every 50-100 yr (Duerbeck \& Seitter 1987). We estimated the age of the shell in Section 3.3.5 as 500-1200 yr, so the original ejected mass of the shell would have been substantially lower than the value we have derived above, giving a maximum ejected mass of $\lesssim 10^{-5}$ $\mathrm{M}_{\odot}$. This rules out a planetary nebula origin, which typically have masses in the range 0.1-1.0 $\mathrm{M}_{\odot}$ (Osterbrock 1989). Nova shells typically have masses in the range $10^{-4}-10^{-6} \mathrm{M}_{\odot}$ (Yaron et al.
2005), so our estimate of the shell mass in V1315 Aql of $\sim 10^{-5}$ $\mathrm{M}_{\odot}$ is in accordance with this.

\section{DISCUSSION}

We can summarize our findings as follows. The shell is broadly spherical and appears to be centred on V1315 Aql, strongly suggesting that the shell is associated with the central binary. The systemic velocity of the shell measured from the sky portion of the V1315 Aql slit and at the edges of the shell are broadly consistent. The absence of $22 \mu \mathrm{m}$ emission precludes a planetary nebula origin (Mizuno et al. 2010). We derive an order-of-magnitude estimate of the mass of the shell of $\sim 10^{-5} \mathrm{M}_{\odot}$ which rules out a planetary nebula or supernova origin. We conclude that these results indicate that the shell is associated with V1315 Aql.

At this stage of the shell's evolution, the luminosity of the outer edges of the shell is most likely fuelled by two processes, recombination and shock interaction with pre-existing CSM. Our flux measurement will include contributions from both of these processes, making it difficult to estimate the physical conditions in the shell as a whole. Furthermore, the lack of other forbidden emission lines in the shell spectra, especially N[II] $6583 \AA$ and O[II], means we cannot determine the physical parameters of the shell to confirm conclusively that it exhibits properties consistent with a nova origin.

In $\mathrm{S} 15$, we estimated that the nova-like phase following a nova eruption lasts $\sim 2400 \mathrm{yr}$. This is comparable to the $\sim 2000$ yr orderof-magnitude estimate by Patterson et al. (2013), based on the transition of BK Lyn to a dwarf nova in the year 2011. However, the AAVSO light curve of BK Lyn ${ }^{4}$ suggests that it has now reverted back to a nova-like state, indicating that the object is a $\mathrm{Z}$ Cam-type dwarf nova that has likely been transitioning from the nova-like to dwarf nova state for much less than the $\sim 2000$-yr estimate of Patterson et al. (2013). Shara et al. (2017a) found that the transition time for AT Cnc was much shorter at $330_{-90}^{+135}$ yr. Our estimate of the time since the nova eruption on V1315 Aql of 500-1200 yr is consistent with both these time-scales, and lies within the overall nova recurrence time-scale of $13000 \mathrm{yr}$ found by Schmidtobreick et al. (2015).

\footnotetext{
${ }^{4}$ https://www.aavso.org/
} 


\section{CONCLUSIONS}

We present images and spectra of the shell surrounding V1315 Aql. Our results strongly suggest that the shell originated from a nova eruption on the CV. This discovery of the first nova shell around a nova-like variable adds further support to the theory of nova-induced cycles in the mass transfer rates of CVs.

\section{ACKNOWLEDGEMENTS}

We would like to thank the referee for his helpful comments and for pointing out the latest AAVSO light curve of BK Lyn demonstrating Z Cam-like behaviour. We acknowledge with thanks the variable star observations from the AAVSO International Database, contributed by observers worldwide and used in this research.

VSD and SPL were supported under grants from the Science and Technology Facilities Council (STFC). This publication makes use of VOSA, developed under the Spanish Virtual Observatory project supported from the Spanish Ministerio de Ciencia e Innovacion through grant AyA2011-24052. The INT is operated on the island of La Palma by the Isaac Newton Group of Telescopes in the Spanish Observatorio del Roque de los Muchachos of the Instituto de Astrofísica de Canarias. Some of the data presented herein were obtained at the W.M. Keck Observatory, which is operated as a scientific partnership among the California Institute of Technology, the University of California, and the National Aeronautics and Space Administration. The Observatory was made possible by the generous financial support of the W.M. Keck Foundation. The authors wish to recognize and acknowledge the very significant cultural role and reverence that the summit of Mauna Kea has always had within the indigenous Hawaiian community. We are most fortunate to have the opportunity to conduct observations from this mountain.

\section{REFERENCES}

Ak T., Bilir S., Ak S., Eker Z., 2008, New Astron., 13, 133

Bayo A., Rodrigo C., Barrado Y Navascués D., Solano E., Gutiérrez R., Morales-Calderón M., Allard F., 2008, A\&A, 492, 277

Bianchi L., 2014, Ap\&SS, 354, 103

Bode M. F., Evans A., 2008, Classical Novae, 2nd edn. Cambridge Univ.

Press, Cambridge

Büning A., Ritter H., 2004, A\&A, 423, 281
Corradi R. L. M., García-Rojas J., Jones D., Rodríguez-Gil P., 2015, ApJ, 803,99

Dhillon V. S., Rutten R. G. M., 1995, MNRAS, 277, 777

Dhillon V. S., Marsh T. R., Jones D. H. P., 1991, MNRAS, 252, 342

Downes A. R., Matteo M., Szkody P., Jenner D. C., Margon B., 1986, ApJ, 301,240

Downes R. A., Duerbeck H. W., Delahodde C. E., 2001, J. Astron. Data, 7 , 6

Duerbeck H. W., Seitter W. C., 1987, Ap\&SS, 131, 467

Faber S. M. et al., 2003, in Iye M., Moorwood A. F. M., eds, Proc. SPIE Conf. Ser. Vol. 4841, Instrument Design and Performance for Optical/Infrared Ground-based Telescopes. SPIE, Bellingham, p. 1657

Hardy L. K., Butterley T., Dhillon V. S., Littlefair S. P., Wilson R. W., 2015, MNRAS, 454, 4316

Knigge C., 2010, in Kologera V., van der Sluys M., eds, AIP Conf. Proc. Vol. 1314, Cataclysmic Variables: Eight Breakthroughs in Eight Years, Am. Inst. Phys., New York, p. 171

Knigge C., Baraffe I., Patterson J., 2011, ApJS, 194, 28

Mizuno D. R. et al., 2010, AJ, 139, 1542

Osaki Y., 1974, PASJ, 26, 429

Osterbrock D. E., 1989, Astrophysics of Gaseous Nebulae and Active Galactic Nuclei. University Science Books, Sausolito, CA

Patterson J. et al., 2013, MNRAS, 434, 1902

Rodríguez-Gil P. et al., 2007, MNRAS, 377, 1747

Rutten R. G. M., van Paradijs J., Tinbergen J., 1992, A\&A, 260, 213

Sahman D. I., Dhillon V. S., Knigge C., Marsh T. R., 2015, MNRAS, 451, 2863, (S15)

Schmidtobreick L., Shara M., Tappert C., Bayo A., Ederoclite A., 2015 , MNRAS, 449, 2215

Shara M. M., Livio M., Moffat A. F. J., Orio M., 1986, ApJ, 311, 163

Shara M. M. et al., 2007, Nature, 446, 159

Shara M. M., Mizusawa T., Wehinger P., Zurek D., Martin C. D., Neill J. D., Forster K., Seibert M., 2012, ApJ, 758, 121

Shara M. M., Drissen L., Martin T., Alarie A., Stephenson F. R., 2017a, MNRAS, 465, 739

Shara M. M. et al., 2017b, Nature, 548, 558

Skrutskies M. F. et al., 2006, ApJ, 131, 1163

Stephenson F. R., 1976, QJRAS, 17, 121

Warner B., 1995, Cataclysmic Variable Stars. Cambridge Univ. Press, Cambridge

Wright E. L. et al., 2010, AJ, 140, 1868

Yaron O., Prialnik D., Shara M. M., Kovetz A., 2005, ApJ, 623, 398

This paper has been typeset from a $\mathrm{T}_{\mathrm{E}} \mathrm{X} / \mathrm{L} \mathrm{T} \mathrm{E} \mathrm{X}$ file prepared by the author. 